J. Lake Sci.(湖泊科学), 2018, 30(1): 91-101

DOI 10. 18307/2018. 0109

(C) 2018 by Journal of Lake Sciences

\title{
太湖藻源溶解性有机质光化学降解研究”
}

\author{
孙 伟 ${ }^{1,2}$, 巩小 丽 $^{2}$, 陈 显 ${ }^{2,3}$, 郑建伟 ${ }^{1}$, 李 琪 $^{1 * *}$, 杜瑛珣 ${ }^{2 * *}$ \\ ( 1 : 南京信息工程大学应用气象学院,南京 210044) \\ (2: 中国科学院南京地理与湖泊研究所湖泊与环境国家重点实验室,南京 210008) \\ (3: 云南大学国际河流与生态安全研究院, 昆明 650031)
}

摘 要: 蓝藻水华暴发过程会产生大量的溶解性有机质一一藻源溶解性有机质 (A-DOM); A-DOM 的光化学降解影响其 迁移转化和在湖泊中的功能. 本研究从太湖藻华中提取 A-DOM, 利用三维苂光光谱一平行因子分析法 (EEMsPARAFAC), 研究 A-DOM 中各组分的光化学降解; 再研究不同光照强度、溶解氧浓度、A-DOM 浓度、波长对 A-DOM 的降 解的影响. 结果显示, A-DOM 中含有 4 种 EEMs-PARAFAC 组分: C1 ( UVC 类腐殖质)、C2 (UVA 类腐殖质)、C3 (类色氨 酸) 和 $\mathrm{C} 4$ (类络氨酸), 对总苂光强度的贡献比例分别为 $22.2 \% 、 8.6 \% 、 68.1 \%$ 和 $1.1 \%$. 当 DOC 初始浓度为 $10 \mathrm{mg} / \mathrm{L}$ 、反应 温度为 $28^{\circ} \mathrm{C} 、 \mathrm{pH}=8.0$ 时, 经 $500 \mathrm{~W}$ 永灯 $\left(391.7 \mathrm{~W} / \mathrm{m}^{2}\right.$ ) 光照 $12 \mathrm{~h}, \mathrm{~A}-\mathrm{DOM}$ 的总光化学降解率 (以 $a_{355}$ 计) 为 $70.4 \%$; 苂光组 分 C1、C2 和 C3 的降解率分别为 $96.1 \% 、 85.4 \%$ 和 $99.2 \%$, 三者的光反应性为 C3>C1>C2. 条件控制实验显示溶解氧的增加 和光强的增强均有助于 A-DOM 的降解; A-DOM 光化学降解主要发生在紫外区, 可见光不能使 C1 和 C2 得到降解. 结果表 明 A-DOM 的光化学降解速度较快, 且能通过控制光强、照射光波长和引人溶解氧等条件控制降解速度. 实验结果可为湖 泊蓝藻水华暴发时的应急处理和保障饮用水安全提供理论依据.

关键词: 蓝藻; 水华;光化学降解;三维苂光光谱一平行因子分析 ;太湖

\section{Photochemical degradation of the algae-derived dissolved organic matter in Lake Taihu}

\author{
SUN Wei ${ }^{1,2}$, GONG Xiaoli ${ }^{2}$, CHEN Yu ${ }^{2,3}$, ZHENG Jianwei ${ }^{1}$, LI Qi $^{1 * *}$ \& DU Yingxun ${ }^{2 * *}$ \\ (1: College of Applied Meteorology, Nanjing University of Information Science and Technology, Nanjing 210044, P.R. China) \\ (2: State Key Laboratory of Lake Science and Environment, Nanjing Institute of Geography and Limnology, Chinese Academy \\ of Sciences, Nanjing 210008, P.R. China) \\ (3: Institute of International Rivers and Eco-Security, Yunnan University, Kunming 650031, P.R.China)
}

\begin{abstract}
During the cyanobacteria bloom, a large amount of dissolved organic matter could be produced. The photochemical degradation is one important transformation way of algae-derived dissolved organic matter (A-DOM). In this study, the photodegradation of A-DOM, extracted from the blooming cyanobacteria in Lake Taihu, is investigated by UV-Vis spectrum and excitation emission matrix spectroscopy combined with parallel factor analysis (EEMs-PARAFAC). In addition, the effects of the light intensity, light wavelength, dissolved oxygen (DO) concentration and the initial A-DOM concentration on the photodegradation are studied. Four PARAFAC components are identified, including C1 (UVC humic-like substance), C2 (UVA humic-like substance), C3 (tryptophan-like substance) and C4 (tyrosine-like substance) with the relative abundances of $22.2 \%, 8.6 \%, 68.1 \%$ and $1.1 \%$, respectively. Under $12 \mathrm{~h}$ radiation by a $500 \mathrm{~W}$ mercury lamp, the removal percentages of $a_{355}, \mathrm{C} 1, \mathrm{C} 2$ and $\mathrm{C} 3$ are $70.4 \%$, $96.1 \%, 85.4 \%$ and $99.2 \%$, respectively $\left(\mathrm{pH}=8.0 ; \mathrm{T}=28^{\circ} \mathrm{C} ;[\mathrm{DOC}]_{0}=10 \mathrm{mg} / \mathrm{L}\right)$. The photoreactivities of the three PARAFAC components follows $\mathrm{C} 3>\mathrm{C} 2>\mathrm{C} 1$. Increase in the light intensity and DO concentration is helpful for the photodegradation of A-DOM. The increase of UV light contributes most photodegradation of A-DOM. The results show that the photodegradation of A-DOM is quick and could be controlled by changing photoreaction operational parameters, which could shed light on the treatment of algae bloom and ensure the safety of drinking water.
\end{abstract}

* 国家自然科学基金项目 (41371122, 41671099)资助. 2016-11-07 收稿; 2017-05-04 收修改稿. 孙伟( 1992 ), 男,硕士研究生; E-mail: 852248278@qq.com.

** 通信作者; E-mail: 363440130@ qq.com,yxdu@ niglas.ac.cn. 
Keywords: Cyanobacteria; blooms; photochemical degradation; EEMs-PARAFAC; Lake Taihu

氮、磷等营养盐的过度输人引起的水体富营养化是世界上最广泛关注的水环境问题之一. 营养盐的输 人导致浮游植物的异常增殖, 出现蓝藻水华. 藻华消退过程会产生大量的溶解性有机质 (称为藻源溶解性有 机质, algae derived-dissolved organic matter, A-DOM $)^{[1]}$, A-DOM 很快就会消耗水体中的溶解氧 (DO), 使得厌 氧条件下藻类代谢产生二甲基异茨醇等恶臭物质, 严重危及到人们饮用水安全 ${ }^{[2]}$. 因此, 加快 A-DOM 的降 解, 有利于消除藻华暴发对饮用水安全的不利影响. 光化学降解是 DOM 重要的降解途径; 相对于生物降解, 光化学降解的速度更快 ${ }^{[3]}$.

由于 DOM 成分复杂, 目前很难以对其进行全面的分析. 近年来发展起来的三维苂光光谱一平行因子分 析法 (EEMs-PARAFAC) 能够很好区分 DOM 的组分和来源 ${ }^{[4]}$. 利用 EEMs-PARAFAC 技术, 可以研究各 EEMs-PARAFAC 组分的光反应性和其在反应过程中的变化 ${ }^{[5-10]}$. 但是, 在不同研究中, 一些相似组分却表现 出不同的光反应性. 例如 Stedmon 等 ${ }^{[11]}$ 研究了浮游植物群落产生的 DOM 的光降解, 发现类色氨酸 (类蛋白 质的一种) 能被光降解; 但张运林等 ${ }^{[12]}$ 认为类蛋白质难以被光降解. 以上研究的不一致可能在于实验条件 的差异, Stedmon 采用了人工光源, 而张运林等利用自然光照射; 且两个研究中 DOM 的 EEMs-PARAFAC 组 分有细微差异.

可见, 反应条件对 DOM 的光化学降解有着重要的影响; 有机物的光化学降解过程可能受各种条件 (包 括光源、光强、 $\mathrm{pH}$ 、载气等) 的影响 ${ }^{[13-14]}$. 但目前, 关于反应条件对 A-DOM 的 EEMs-PARAFAC 组分光化学降 解的影响方面的研究较少. 明确 A-DOM 的降解效率的影响因素能为消除藻华暴发对饮用水的影响提供理 论依据; 也有助于了解 A-DOM 的迁移转化过程. 因此, 本文以 A-DOM 为研究对象, 利用 EEMs-PARAFAC, 研 究 A-DOM 的光化学降解; 并通过控制不同实验条件 (光照强度、照射光波长、DOC 浓度和载气), 来研究各 参数对 A-DOM 降解的影响. 结果揭示了 A-DOM 各组分的光反应性及不同条件对其光化学降解的影响, 有 助于了解 A-DOM 的迁移转化, 能为湖泊蓝藻水华暴发时应急处理和保障饮用水安全提供依据.

\section{1 材料与方法}

\subsection{A-DOM 的提取}

于 2016 年 6 月 14 日在中国科学院太湖湖泊生态系统试验站 $\left(31^{\circ} 25.42^{\prime} \mathrm{N}, 120^{\circ} 12.57^{\prime} \mathrm{E}\right)$ 采集含蓝藻水 样 $10 \mathrm{~L}$, 再通过浮游植物网过滤浓缩得到含蓝藻浓度较高的水样; 对浓缩后的藻水进行离心 (9000 转/ min, $15 \mathrm{~min}$ ), 分离出藻浆; 然后将藻浆放人超纯水中混合清洗, 再离心出藻浆, 重复 3 遍; 最后用液氮将蓝藻快速 冷冻, 再解冻, 重复 3 遍, 将蓝藻细胞打破 ${ }^{[15]}$. 处理后的溶液经孔径为 $0.22 \mu \mathrm{m}$ 的滤膜 ( Millipore, PES Membrane) 过滤得到 A-DOM 溶液, 此溶液为 A-DOM 的储备液, 冷藏. 经测定, 储备液浓度为 $550 \mathrm{mg} \mathrm{C} / \mathrm{L}$.

\section{$1.2 \mathrm{~A}-\mathrm{DOM}$ 的光降解}

光化学降解反应在室温条件下的多试管搅拌型光反应仪 (南京胥江, 图 1a) 中进行. 激发光源采用 500 $\mathrm{W}$ 和 $300 \mathrm{~W}$ 的中压录灯, 其发射光谱见图 $1 \mathrm{~b}$; 根据发射光谱图计算, 光强分别为 391.7 和 $91.5 \mathrm{~W} / \mathrm{m}^{2}$. 在紫 外和可见光波段光强分别为 $140.3 、 251.4 \mathrm{~W} / \mathrm{m}^{2}$ ( $500 \mathrm{~W}$ 永灯), $30.2 、 61.3 \mathrm{~W} / \mathrm{m}^{2}$ ( $300 \mathrm{~W}$ 录灯); 反应前将一 定体积的 A-DOM 储备液配制成 $500 \mathrm{ml} 10 \mathrm{mg} \mathrm{C} / \mathrm{L}$ 的溶液; 使用 $0.1 \mathrm{~mol} / \mathrm{L}$ 的 $\mathrm{HCl}$ 和 $0.1 \mathrm{~mol} / \mathrm{L}$ 的 $\mathrm{NaOH}$ 调节 $\mathrm{pH}$; 将每 $50 \mathrm{ml}$ 配制好的溶液加人 9 个石英反应管中, 开启冷却水装置, 控制温度为 $28^{\circ} \mathrm{C}$ 左右; 待系统稳定 后, 打开录灯光源, 以此作为反应起点. 在特定反应时间 $(0 、 0.5 、 1 、 2 、 4 、 6 、 8 、 10 、 12 \mathrm{~h})$ 各取出一个石英反应 管. 取出的水样放人冰箱中冷藏, 在两周内进行紫外一可见光全波扫描 (UV-Vis)、三维苂光光谱 (EEMs) 分析.

通人载气 $\left(\mathrm{O}_{2}\right.$ 或者 $\left.\mathrm{N}_{2}\right)$ 实验, 在开启冷却水后, 往反应管中通人 $\mathrm{O}_{2}$ 或者 $\mathrm{N}_{2} 30 \mathrm{~min}$, 使得溶液中 DO 接近 饱和或约为 0 ; 再开启永灯启动实验; 实验过程一直通人载气.

\section{$1.3 \mathrm{~A}-\mathrm{DOM}$ 的分析}

紫外一可见光全波扫描光谱 (UV-Vis Spectra) : 使用 Lambda 35 (Perkin Elmer) 紫外可见分光光度计, 测 试范围为 200 $800 \mathrm{~nm}$, 参比为 Milli-Q 水. 根据式(1) 和(2), 通过一定波长下的吸光度计算得到该波长下的 

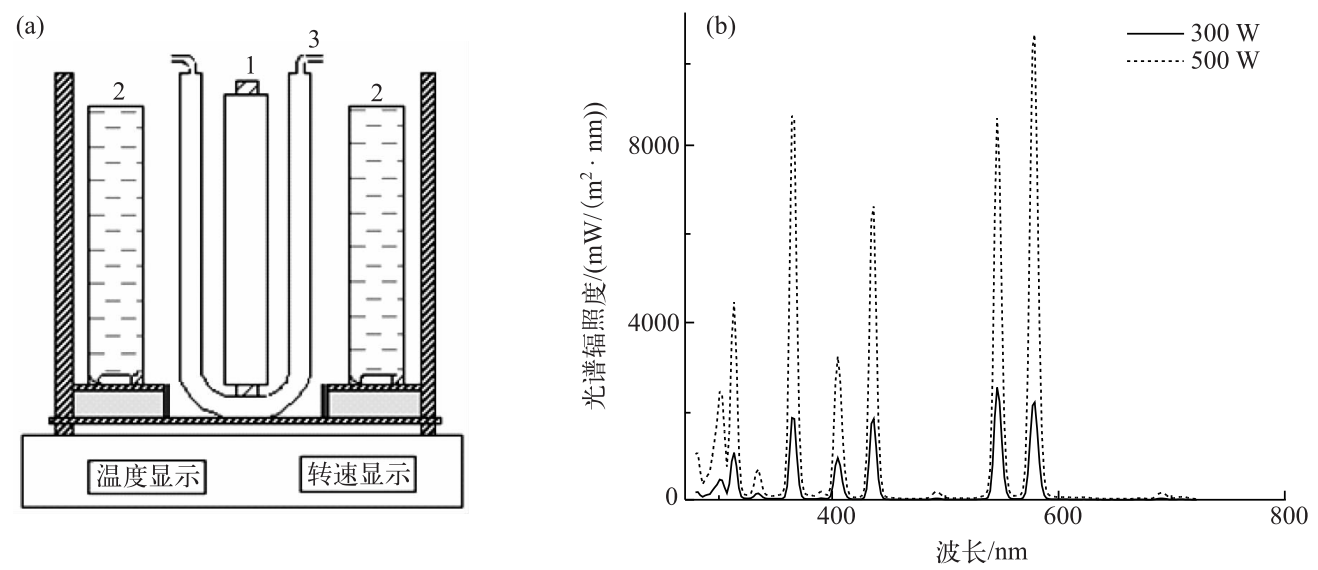

图 1 光降解反应器示意图 (a) ( 1 : 光源; 2 : 石英反应管; 3 : 石英冷阱) 及中压永灯发射光谱图 (b)

Fig.1 The schematic of photodegradation reaction instrument(a) (1-lamp; 2-water-cooled jacket; 3 -quartz tube) and the emission spectrum of median-pressure spectrum of mercury lamp (b)

吸收系数 ${ }^{[16]}$ :

$$
a\left(\lambda^{\prime}\right)=2.303 D(\lambda) / r
$$

式中, $a\left(\lambda^{\prime}\right)$ 为波长 $\lambda$ 下未校正的吸收系数 $\left(\mathrm{m}^{-1}\right) ; D(\lambda)$ 为吸光度 $; r$ 为光程路径 $(0.01 \mathrm{~m})$. 由于过滤清液 可能残留细小颗粒会引起散射,为此作如下散射效应订正 ${ }^{[17]}$ :

$$
a(\lambda)=a\left(\lambda^{\prime}\right)-a(750) \lambda / 750
$$

式中, $a(\lambda)$ 为波长 $\lambda$ 的吸收系数 $\left(\mathrm{m}^{-1}\right) ; \lambda$ 为波长 $(\mathrm{nm})$.

采用光谱斜率比值 $\left(S_{\mathrm{R}}\right)$ 表征 A-DOM 分子量的变化, $S_{\mathrm{R}}$ 与相对分子量呈反比 ${ }^{[18-19]}$, 其计算方法为:

$$
S_{\mathrm{R}}=S_{(275-295)} / S_{(350-400)}
$$

式中, $S_{(275-295)}$ 和 $S_{(350-400)}$ 分别为波长 $275 \sim 295 \mathrm{~nm}$ 和 $350 \sim 400 \mathrm{~nm}$ 的光谱斜率 $\left(\mathrm{nm}^{-1}\right)$.

EEMs 采用日立 F-7000 苂光光谱仪测定 (Hitachi High-Technologies). 激发波长为 $200 \sim 450 \mathrm{~nm}$, 以 $5 \mathrm{~nm}$ 为间隔; 发射波长为 $250 \sim 550 \mathrm{~nm}$, 以 $1 \mathrm{~nm}$ 为间隔. 仪器校正根据日立 F-7000 说明书进行 ${ }^{[20]}$. EEMsPARAFAC 模型的建立: 首先对三维光谱进行校正: (1) 根据 McKnight 等利用紫外一可见吸收光谱数据进行 内滤效应修正 ${ }^{[21]}$; (2) 用 Milli-Q 水做空白扣除; (3) 利用 Milli-Q 水的拉曼单位归一化 (R.U.) 来校准日常的 苂光强度的变化. 再参考 Stedmon 等 ${ }^{[22]}$ 提出的方法, 利用 DOMFluor 工具箱在 MATLAB R2008a 软件中得到 4 个组分模型 (C1 C4), 通过折半分析 (split-half analysis) 和随机初始化 (random initialization) 方法来验证模 拟结果的可靠性, 识别出 4 个组分的苂光强度.

此外利用拟一级降解动力学方程 (式 (4)) 来确定各个 PARAFAC 组分的降解速率 ${ }^{[23]}$ :

$$
\ln C / C_{0}=-\mathrm{k} \cdot t+\mathrm{A}
$$

式中, $C$ 为某一光照时间 PARAFAC 组分的苂光强度 (R.U.); $C_{0}$ 为该组分的初始苂光强度 (R.U.); $t$ 为光照 时间 $(h) ; k$ 为拟一级降解动力学常数 $\left(h^{-1}\right), k$ 值越大表示光化学降解率越快; $A$ 为常数.

\section{2 结果与讨论}

\subsection{A-DOM 的 EEMs-PARAFAC 组分}

根据不同光照条件实验获得的 EEMs 数据, 建立了 4 个组分 $(\mathrm{C} 1 \sim \mathrm{C} 4)$ 模型. 其三维苂光光谱图及激发、 发射波长特征图见图 2. 表 1 列出了 $\mathrm{C} 1 \sim \mathrm{C} 4$ 激发和发射波长峰值及分类.

$10 \mathrm{mg} \mathrm{C} / \mathrm{L}$ 的 A-DOM 中 $\mathrm{C} 1 \sim \mathrm{C} 4$ 的苂光强度分别为 $2.13 、 0.82 、 6.52$ 和 0.11 R.U., 各组分在 A-DOM 苂光 贡献百分比分别是 $22.2 \% 、 8.6 \% 、 68.1 \%$ 和 $1.1 \%$, 说明 A-DOM 的荧光贡献中, 以含类色氨酸基团的组分为 主, 还有部分 UVA、UVC 类腐殖质. 

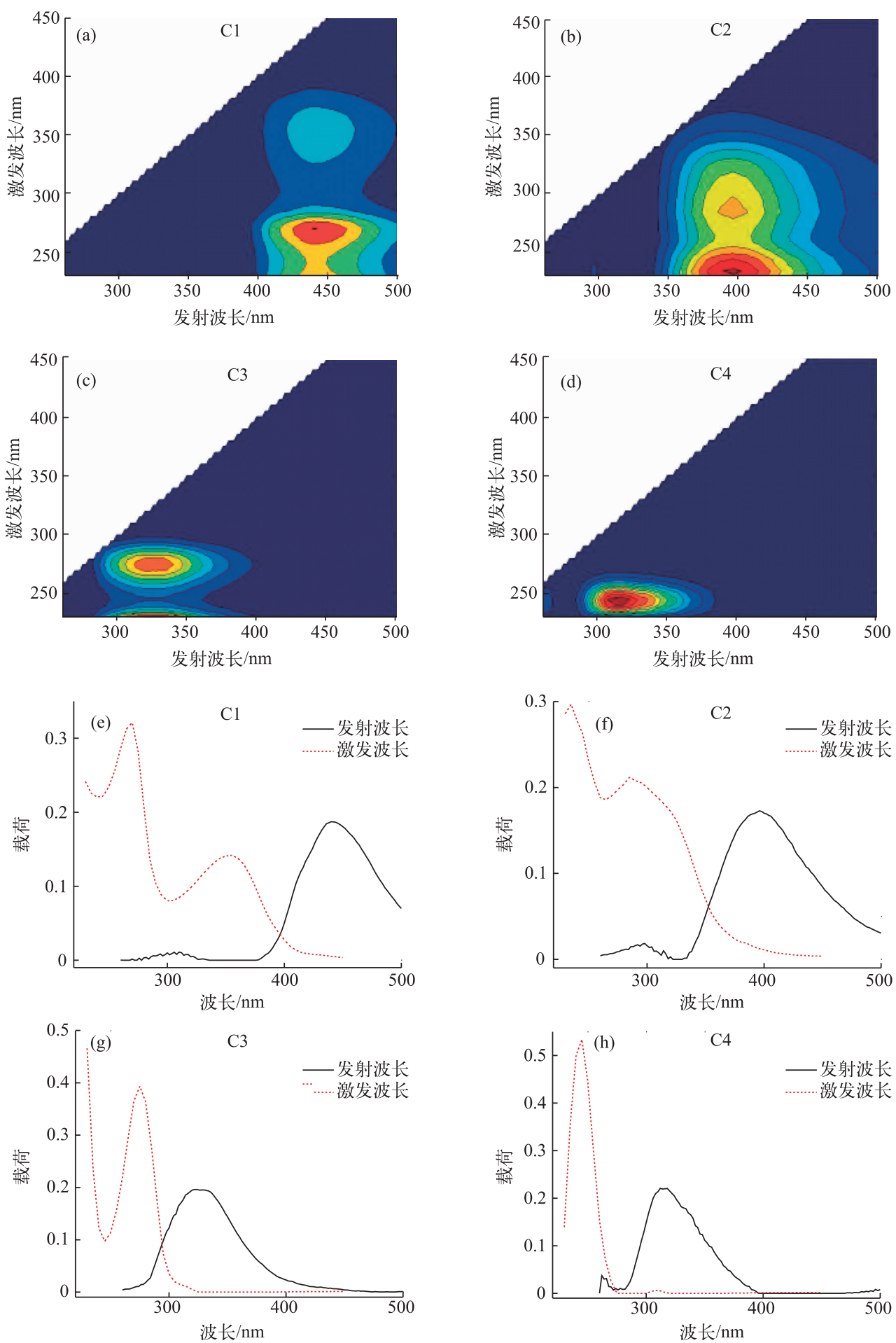

图 2 平行因子分析得到的 4 个组分三维菼光光谱图 $(\mathrm{a} \sim \mathrm{d})$ 及激发、发射波长特征图 $(\mathrm{e} \sim \mathrm{h})$

Fig.2 EEMs contours $(\mathrm{a}-\mathrm{d})$ and the spectral characteristics of the EEMs-PARAFAC components $(\mathrm{e}-\mathrm{h})$ 
表 $1 \mathrm{C} 1 \sim \mathrm{C} 4$ 激发和发射波长峰值及分类

Tab.1 Excitation and emission maxima of the four PARAFAC components and possible assignments

\begin{tabular}{|c|c|c|c|c|}
\hline 组分 & 激发波长峰值/nm & 发射波长峰值/nm & 分类 & 对总苂光强度的贡献比例 \\
\hline C1 & $270(355)$ & 440 & UVC 类腐殖质 [24-27] & $22.2 \%$ \\
\hline $\mathrm{C} 2$ & $235(285)$ & 396 & UVA 类腐殖质 ${ }^{[25-28]}$ & $8.6 \%$ \\
\hline $\mathrm{C} 3$ & 275 & 322 & 类色氨酸 ${ }^{[25-27]}$ & $68.1 \%$ \\
\hline $\mathrm{C} 4$ & 245 & 312 & 类络氨酸 ${ }^{[26]}$ & $1.1 \%$ \\
\hline
\end{tabular}

\subsection{A-DOM 的光降解行为研究}

从光照过程 A-DOM 的 UV-Vis 光谱变化可以看出, 未经光照 $(0 \mathrm{~h})$ 的 A-DOM 有两个吸收峰, 分别在 260 和 $330 \mathrm{~nm}$; A-DOM 对紫外光吸收较为明显, 而对于可见光几乎不吸收. 随着实验的进行, A-DOM 的吸光度 不断减小, 最大吸收波长的吸光度在 $200 \sim 355 \mathrm{~nm}$ 处下降最为明显 (图 3). 为了定量地描述光化学降解的
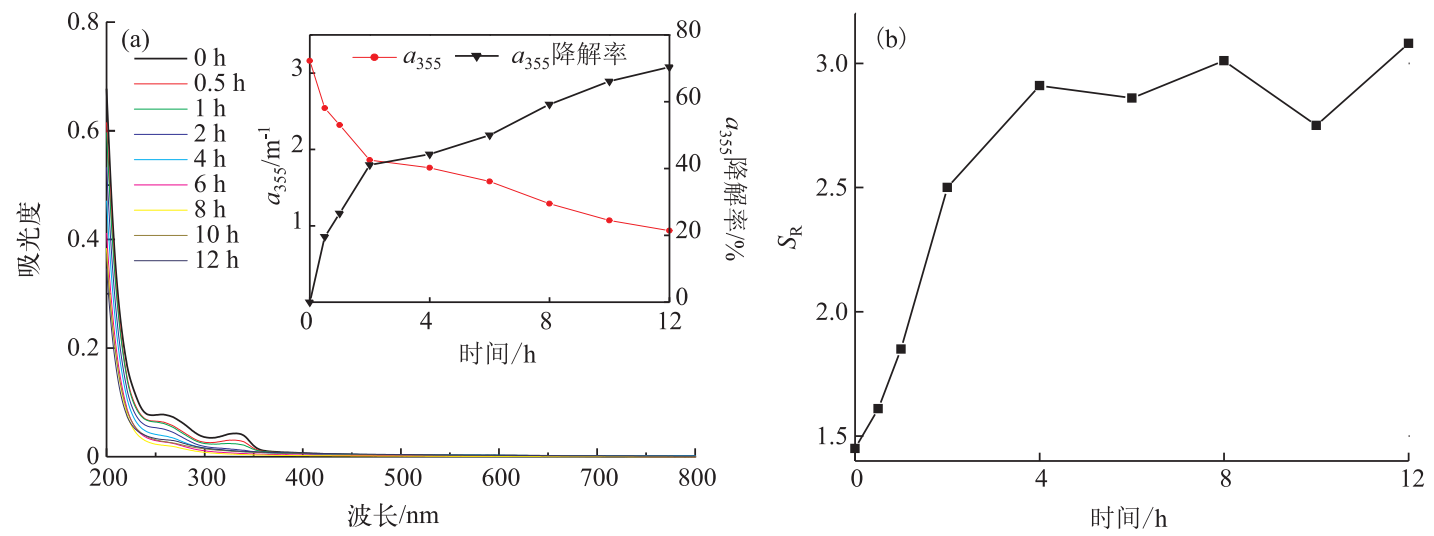

图 3 A-DOM 降解过程 UV-Vis 光谱 (a) (光源 $=500 \mathrm{~W}$ 采灯; 不加滤光膜; $\mathrm{pH}=8.0 ; \mathrm{T}=28^{\circ} \mathrm{C}$;无载气; $\left.[\mathrm{DOC}]_{0}=10 \mathrm{mg} / \mathrm{L}\right)$ 及 $S_{\mathrm{R}}$ 的变化 $(\mathrm{b})$

Fig. 3 Changes of UV-Vis spectra during the photodegradation of A-DOM(a) (500 W mercury lamp; without filtered membrane; $\mathrm{pH}=8.0 ; \mathrm{T}=28^{\circ} \mathrm{C} ;$ no carrier gas; $\left.[\mathrm{DOC}]_{0}=10 \mathrm{mg} / \mathrm{L}\right)$ and spectral slope ratio $(\mathrm{b})$

过程,我们采用 $355 \mathrm{~nm}$ 处的吸收系数 $\left(a_{355}\right)$ 的变化和降 解率 (降解率 $\left.(\%)=\left[1-\left[a_{355}\right] /\left[a_{355}\right]_{0}\right] \times 100\right)$ 来表征 A-DOM 的降解 (图 3a). 可见 $a_{355}$ 在前 $2 \mathrm{~h}$ 内降解速度很 快, 反应初始 $a_{355}$ 为 3.16 , 经过 2 个小时的光化学降解 后吸光系数为 $1.86 \mathrm{~m}^{-1}$, 降解率为 $41.1 \% ; 2 \mathrm{~h}$ 之后, $a_{355}$ 的减少速率变缓; $12 \mathrm{~h}$ 后吸光系数为 $0.94 \mathrm{~m}^{-1}, a_{355}$ 降解 率为 $70.4 \% . S_{\mathrm{R}}$ 在光化学降解的前 $4 \mathrm{~h}$ 内由开始的 1.45 迅速上升至 2.91 , 之后逐渐趋于平缓, 说明光化学降解 的过程中 A-DOM 的分子量会逐渐减小, 大分子 DOM 裂 解为小分子 DOM (图 3b).

为了更好地了解光化学降解各组分的变化过程, 我 们将 EEMs-PARAFAC 模型的 3 个组分 (C1 C3) 作图分 析 (图 4). 可以看出, C1 C3 组分在光照过程中呈下降 趋势. 对比 $\mathrm{C} 1 、 \mathrm{C} 2$ 和 $\mathrm{C} 3$ 的降解, 类蛋白质组分 $\mathrm{C} 3$ 的降 解速率最快, 经 $0.5 \mathrm{~h}$ 的光照, 其降解率为 $89 \%$; 在整个

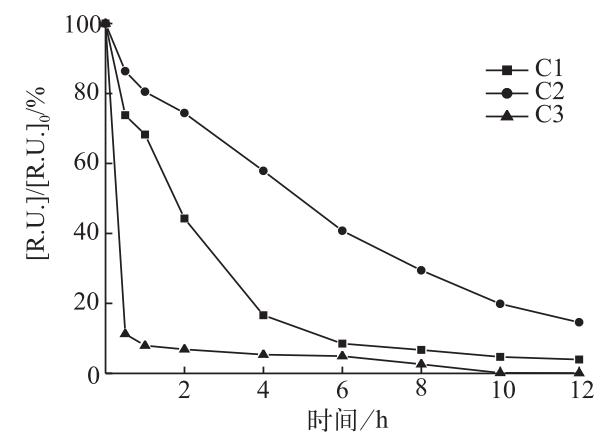

图 4 光化学降解过程 $\mathrm{C} 1 、 \mathrm{C} 2$ 和 $\mathrm{C} 3$ 组分的变化 （光源 $=500 \mathrm{~W}$ 永灯; 不加滤光膜; $\mathrm{pH}=8.0$; $\mathrm{T}=28^{\circ} \mathrm{C}$;无载气; $[\mathrm{DOC}]_{0}=10 \mathrm{mg} / \mathrm{L}$ )

Fig.4 Changes of C1, C2 and C3 during the photochemical degradation ( $500 \mathrm{~W}$ mercury lamp; without filtered membrane; $\mathrm{pH}=8.0 ; \mathrm{T}=28^{\circ} \mathrm{C}$; no carrier gas; $[\mathrm{DOC}]_{0}=10 \mathrm{mg} / \mathrm{L}$ ) 
光照过程中, 其降解率为 $99.9 \%$, 说明 C3 组分光反应性很强, 极易发生光化学降解; 这和 Stedmon 的报道是 一致的. 对于两种类腐殖组分 $\mathrm{C} 1$ 和 $\mathrm{C} 2$, C 1 组分在反应的前 $4 \mathrm{~h}$ 快速降解, $t=4 \mathrm{~h}$ 时降解率为 $83.4 \%, 4 \mathrm{~h}$ 之 后, 降解速率趋于平缓; $12 \mathrm{~h}$ 时, C1 的降解率为 $96.1 \%$. 相对于 $\mathrm{C} 1, \mathrm{C} 2$ 的降解速率较为缓慢, 整个实验的光 化学降解率为 $85 \%$, 降解过程中呈下降趋势, 未出现明显的先快速降解再缓慢降解的趋势. C1 比 C2 拥有更 长的激发波长; 这与文献 [29]中报道的一致: 有更长激发波长的类腐殖质的光降解速度快于短激发波长的 类腐殖质. 通过拟合出各组分的光化学降解动力学常数, 来分析各组分的的光反应性. C1、C2 和 C3 降解动 力学常数分别为 $0.2848 、 0.1568 、 0.4611\left(R^{2}>0.9\right)$, 降解动力学常数越大其降解速率也就越快, 由此进一步证 明了三者的光反应性为 C3>C1>C2. 由于 C4 (类络氨酸) 初始的菼光强度为 0.109 R.U., 在 A-DOM 的苂光贡 献中占比仅为 $1.1 \%$, 且在后续光化学降解过程中荧光强度变化不大; 在 $12 \mathrm{~h}$ 的光化学降解过程后, 其荧光 强度微微上升至 0.118 R.U., 由此可以推断光照对 C4 组分浓度无较大影响, 因此在光化学降解过程中不加 以分析.

\section{3 光照条件对 A-DOM 光降解的影响}

\section{1 光照强度的影响}

图 5a 显示了不同光强 (500 和 $300 \mathrm{~W}$ 永灯) A-DOM 降解过程 $a_{355}$ 的变化情况, 其纵坐标代表的是不同时 间下 $a_{355}$ 相对于 $\left[a_{355}\right]_{0}$ (初始 $a_{355}$ ) 的比值. 可见, 随着光强的升高, $a_{355}$ 的降解速率加快, 由此推断出 A-DOM 的降解速率加快. 在 500 和 $300 \mathrm{~W}$ 采灯光照条件下, 反应 $12 \mathrm{~h}$ 后 $a_{355}$ 降解率分别为 $70.4 \%$ 和 $53.1 \%$. 由此可
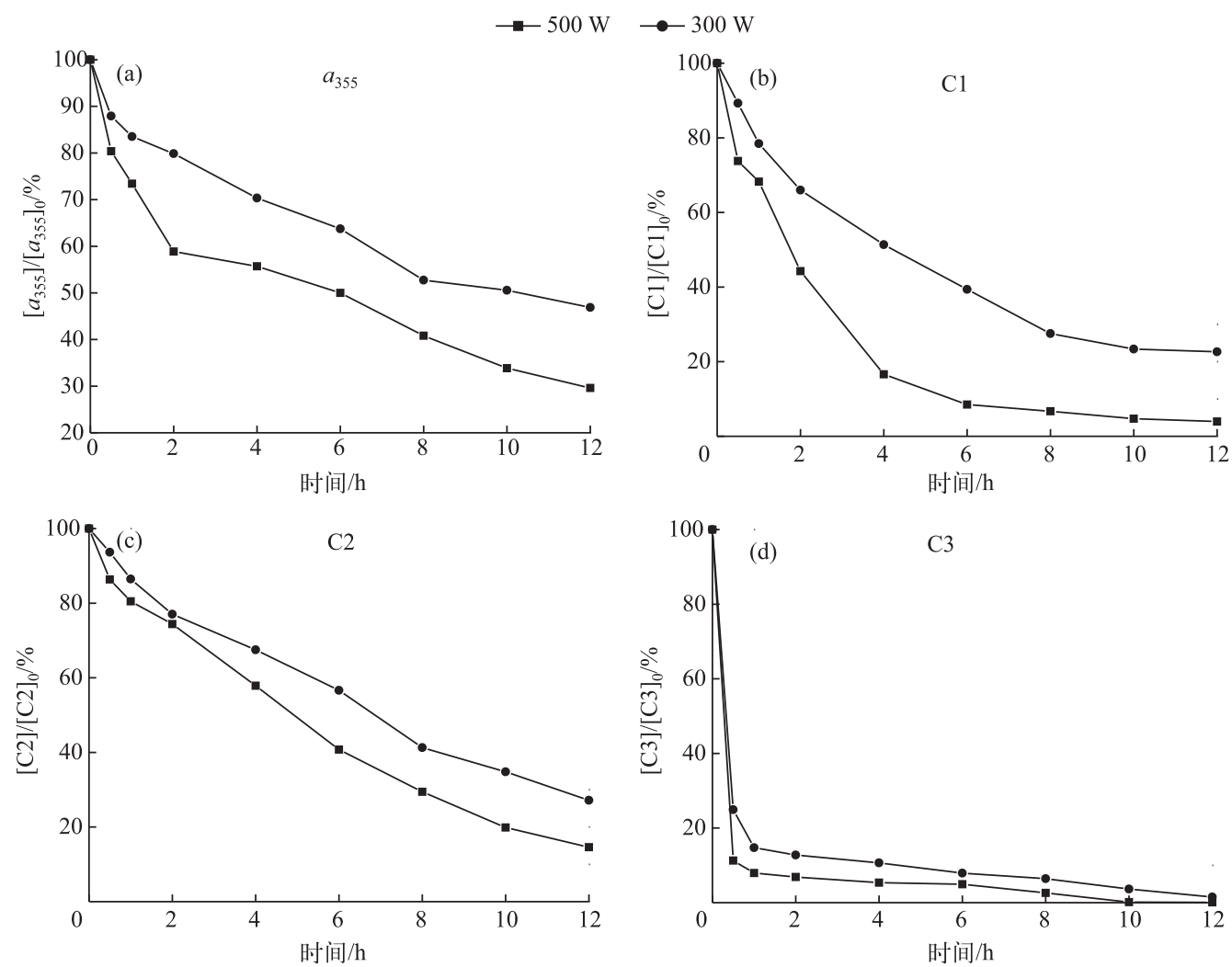

图 5 光照强度对 A-DOM 光降解的影响 (不加滤光膜; $\mathrm{pH}=8.0 ; \mathrm{T}=28^{\circ} \mathrm{C}$; 无载气; [DOC $]_{0}=10 \mathrm{mg} / \mathrm{L}$ )

Fig.5 Effect of light intensity on the photodegradation of A-DOM ( without filtered membrane; $\mathrm{pH}=8.0 ; \mathrm{T}=28^{\circ} \mathrm{C}$; no carrier gas; $[\mathrm{DOC}]_{0}=10 \mathrm{mg} / \mathrm{L}$ ) 
见, 光照强度对于光化学降解有明显的影响, 光照强度越高, 其总的光化学降解速率越快, 降解率越高. 这可 能是随着光强的增大, 提供的光子增多, A-DOM 能吸收的光子增多, 根据光降解原理 ${ }^{[30-31]}$, 更多的 A-DOM 被激发,继而发生光裂解而被降解.

图 5b d 显示了光强对 3 个组分降解的影响, 其纵坐标代表的是不同时间下 C1、C2 和 C3 相对于各组 分初始苂光强度的比值; 每个组分在两种光强下的降解趋势基本一致,但降解速率有明显区别. 随着光强的 减弱, (从 $500 \mathrm{~W}$ 到 $300 \mathrm{~W}$ ), 组分的降解率减少; 在 500 和 $300 \mathrm{~W}$ 采灯光照下, C1 的光化学降解率分别为 $96.1 \%$ 和 $77.4 \%$, 降解动力学常数为 0.2848 和 $0.1301\left(R^{2}>0.9\right)$; C2 光化学降解率为 $85.4 \%$ 和 $72.8 \%$, 降解动 力学常数为 0.1568 和 $0.1055\left(R^{2}>0.9\right)$; C3 光化学降解率为 $99.2 \%$ 和 $98.5 \%$, 降解动力学常数为 0.4611 和 $0.2431\left(R^{2}>0.8\right)$. 由降解动力学常数可知, 在 500 和 $300 \mathrm{~W}$ 永灯光照下, 3 个组分的降解速率均为 C3>C1> C2. 可见, 光强对组分的光反应性并没有影响. 光源为 $500 \mathrm{~W}$ 采灯时, 光强约为 $300 \mathrm{~W}$ 采灯的 4 倍, 但 3 个组 分的降解速率却只增加 1 倍. 这说明随着光强增大, 虽然降解速度提高, 但更多的能量被浪费. 在利用光强 控制 A-DOM 的降解时,需要结合成本和实际情况加以考虑.

\section{2 波长的影响}

为了考察波长对降解的影响,在冷肼的外面包裹不同类型的滤光膜 (滤膜 1 、滤膜 2), 滤膜 1 滤去 UVB 波段 (280 320 nm) 的紫外光, 滤膜 2 滤去 UVB $(280 \sim 320 \mathrm{~nm})$ 和 UVA 波长 $(320 \sim 400 \mathrm{~nm})$ 的紫外光. 无滤 膜、包裹滤膜 1 、包裹滤膜 2 的处理组分别称为 Vis+UVA+UVB、Vis+UVA 和 Vis 处理组.
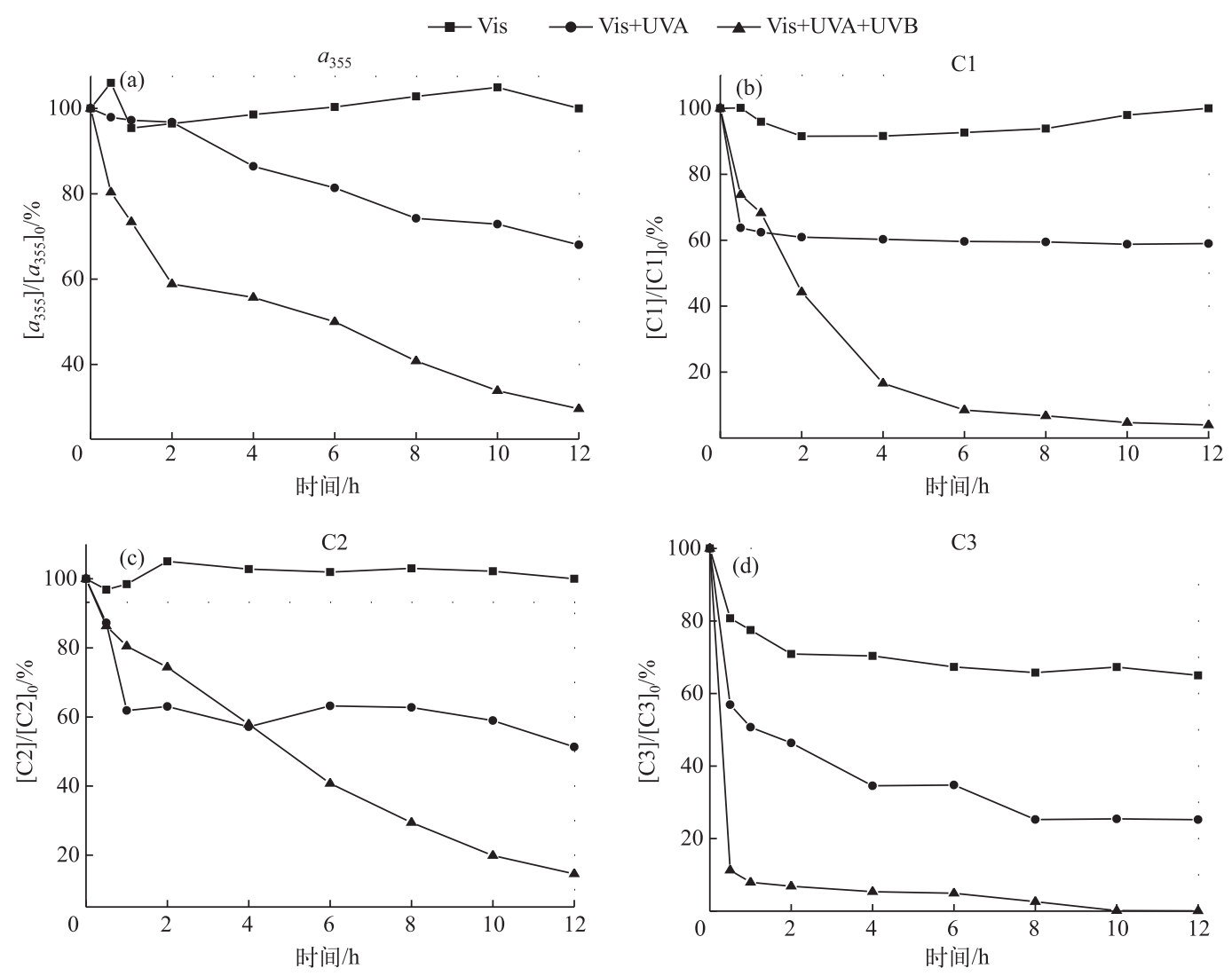

图 6 不同处理组波长对 A-DOM 光降解的影响

$\left(\right.$ 光源 $=500 \mathrm{~W}$ 采灯 $; \mathrm{pH}=8.0 ; \mathrm{T}=28^{\circ} \mathrm{C} ;$ 无载气; $[\mathrm{DOC}]_{0}=10 \mathrm{mg} / \mathrm{L}$ )

Fig.6 Effect of the irradiation wavelength on the photodegradation of A-DOM ( $500 \mathrm{~W}$ mercury lamp; $\mathrm{pH}=8.0 ; \mathrm{T}=28^{\circ} \mathrm{C}$; no carrier gas; $[\mathrm{DOC}]_{0}=10 \mathrm{mg} / \mathrm{L}$ ) 
经 $12 \mathrm{~h}$ 光照后, Vis+UVA+UVB 和 Vis+UVA 处理组中的 $a_{355}$ 降解率分别为 $70.4 \% 、 31.9 \%$, 而 Vis 处理组 中 $a_{355}$ 在误差范围内几乎无变化. A-DOM 在 Vis+UVA+UVB 下光降解速率最快且光化学降解率最高, 在 Vis 处理组几乎没有降解; 由此推断可以使 A-DOM 发生光化学降解的波长主要集中在紫外光区; UV 光的波长 短、能量高, 是光化学降解主要的能量来源光区. 对比 Vis +UVA+UVB 和 Vis $+U V A$ 组, 在滤去 UVB 波长光 后, A-DOM 先降解后趋于平缓不再降解, 说明相对 UVA 光区, UVB 光区对光化学降解的贡献较大 (图 $6 \mathrm{a}$ ). Vis+UVA+UVB、Vis+UVA 和 Vis 处理组 A-DOM 各组分也表现出了类似的规律, C1 的降解率分别为 $96.1 \%$ 、 $41.1 \%$ 和 $2.1 \%$; C2 的降解率分别为 $85.4 \% 、 48.7 \% 、 0.03 \%$; C3 的降解率分别为 $99.9 \% 、 74.8 \%$ 和 32.7\% (图 6b d). 在滤去 UVA 或 UVA+UVB 的处理组, 已不符合一级降解动力学方程, 进一步证明紫外光对各组分的光 化学降解有较大的影响. 通过图 6 可以看出, 在 Vis 处理组中, C1 和 C2 在去除 UV 光后几乎无降解, 而在 $V$ is +UVA+UVB 和 Vis+UVA 处理组均有降解, 由此可以推断出 C1、C2 的光化学降解光区为 UV 区. 在滤去 $\mathrm{UV}$ 光后, $32.7 \%$ 的 $\mathrm{C} 3$ 组分被降解, 由此推断可见光和紫外光均能降解 $\mathrm{C} 3$ 组分, 但紫外光的降解能力更强.

\subsection{A-DOM 浓度的影响}

当初始 DOC 浓度分别为 $5 、 10 、 15$ 和 $20 \mathrm{mg} / \mathrm{L}$ 的 A-DOM 溶液, 经过录灯 $12 \mathrm{~h}$ 的光化学降解, $a_{355}$ 的降解 率分别为 $86.2 \% 、 70.4 \% 、 68.3 \%$ 和 $65.1 \%$ (图 7). 相同条件下 A-DOM 浓度越低越容易降解, 其总的光化学降 解率越高. 当初始 DOC 浓度分别为 5、10、15 和 $20 \mathrm{mg} / \mathrm{L}$ 时, C1 的降解率分别为 $99.4 \% 、 99.2 \% 、 96.8 \%$ 和 $91.1 \%$, 降解动力学常数分别为 $0.4517 、 0.4305 、 0.3824$ 和 $0.3119\left(R^{2}>0.9\right)$; C2 降解率分别为 $96.3 \% 、 91.6 \%$ 、 $85.9 \%$ 和 $73.2 \%$, 降解动力学常数分别为 $0.2998 、 0.1609 、 0.1568$ 和 $0.1114\left(R^{2}>0.9\right)$; C3 降解率分别为 $99.6 \%$ 、
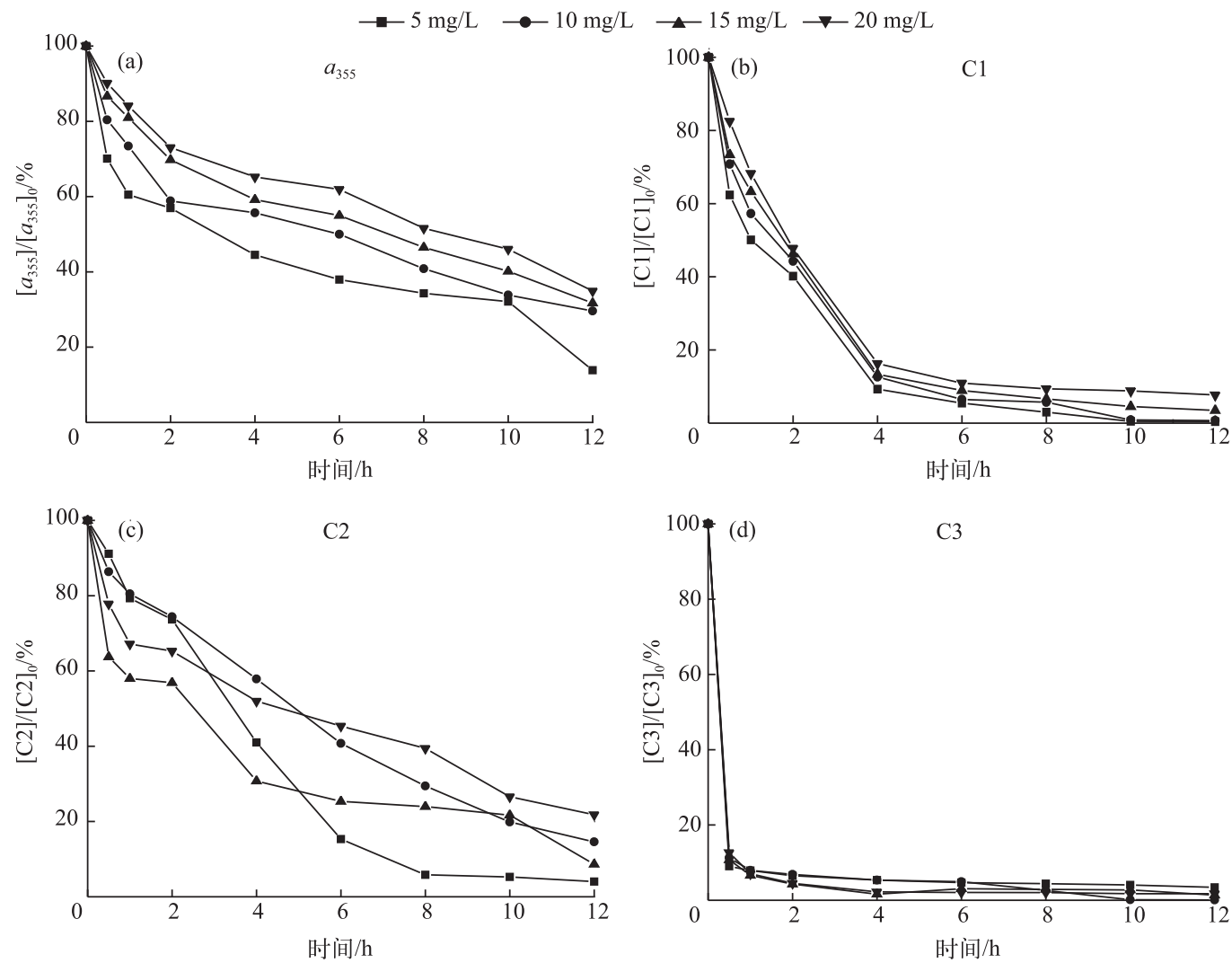

图 7 A-DOM 浓度对其光降解的影响 (光源 $=500 \mathrm{~W}$ 永灯; 不加滤光膜; $\mathrm{pH}=8.0 ; \mathrm{T}=28^{\circ} \mathrm{C}$; 无载气)

Fig.7 Effect of A-DOM concentration on its photodegradation

( $500 \mathrm{~W}$ mercury lamp; without filtered membrane; $\mathrm{pH}=8.0 ; \mathrm{T}=28^{\circ} \mathrm{C}$; no carrier gas) 
$99.3 \% 、 98.7 \%$ 和 $98.3 \%$, 降解动力学常数分别为 $0.4611 、 0.4343 、 0.4079$ 和 $0.354\left(R^{2}>0.8\right)$. 可见, 3 种 PARAFAC 组分中, C2 的光化学降解受浓度的影响最为显著, 浓度越高降解速率越低且降解率也越低; C1 降 解受浓度影响并不是特别明显; C3 组分的光化学降解受浓度的影响最小, 由于 3 组分的光反应性为 C3 $3>\mathrm{C} 1>$ $\mathrm{C} 2, \mathrm{C} 2$ 相对于 C3、C1 光反应性最弱. 当光强一定时, C3 和 C1 对光的能量吸收处于优势, 容易吸收光的能量 发生光解,而被 $\mathrm{C} 2$ 吸收光较少, 因此 C2 受 A-DOM 的浓度影响最为显著.

\section{$3.4 \mathrm{DO}$ 浓度的影响}

通过向光照溶液中通人不同载气的方式来控制水样的 DO 浓度; 通人氧气和氮气,DO 浓度为饱和、接近 于 0 的水平. 不通载气时, DO 浓度处在两者之间. 通氧气、不通载气、通氮气条件下, 经过 $12 \mathrm{~h}$ 的光化学降解 $\mathrm{A}-\mathrm{DOM}$ 的 $a_{355}$ 降解率分别为 $85.6 \% 、 77.4 \% 、 70.4 \%$. 由图 8 可以看出, DO 浓度越高, A-DOM 光化学降解的速 率越快, 且光照 $12 \mathrm{~h}$ 后, 总的光化学降解率也越高. 通过 EEMs-PARAFAC 来分析 DO 对 A-DOM 各组分光化 学降解速率的影响. 在通氧气、不通载气和通氮气的条件下, $12 \mathrm{~h}$ 光照后, C1 组分降解率分别为 $96.3 \%$ 、 $96.1 \%$ 和 $93.1 \%$, 降解动力学常数分别为 $0.2824 、 0.2614$ 和 $0.2094\left(R^{2}>0.9\right)$; C2 组分降解率分别为 $91.2 \%$ 、 $85.5 \%$ 和 $63.4 \%$, 降解动力学常数分别为 $0.1586 、 0.1233$ 和 $0.0769\left(R^{2}>0.9\right)$; C3 组分降解率分别为 $99.9 \%$ 、 $99.9 \%$ 和 $92.8 \%$, 降解动力学常数分别为 $0.4615 、 0.4417 、 0.4303\left(R^{2}>0.7\right)$. 有机物的光化学降解有两个途径, 一是直接光降解, 即溶解性有机质直接吸收光的能量发生的降解变化; 另一个是间接光解, 通过光照过程中
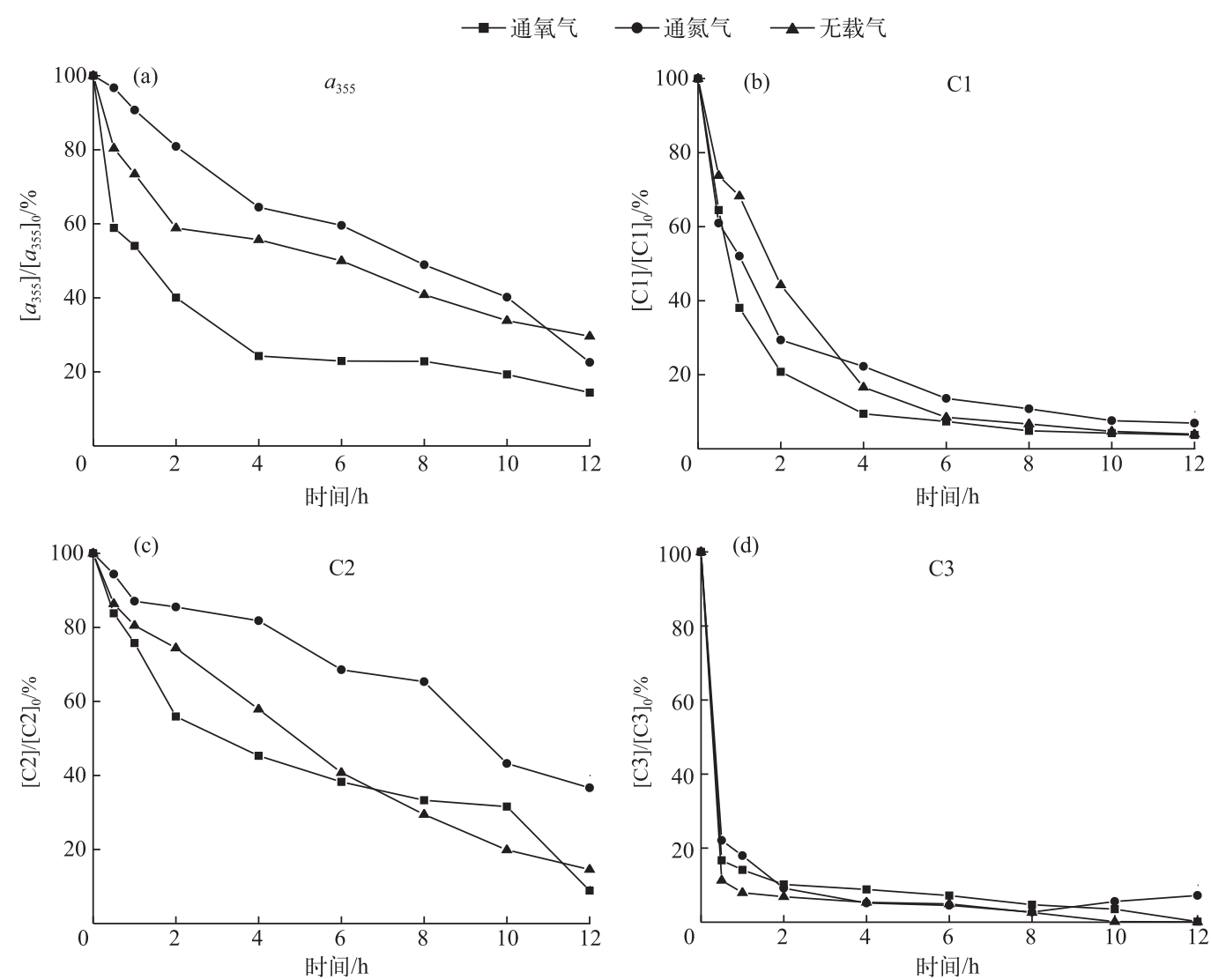

图 $8 \mathrm{DO}$ 浓度对 A-DOM 光降解的影响 (光源 $=500 \mathrm{~W}$ 采灯; 不加滤光膜; $\mathrm{pH}=8.0 ; \mathrm{T}=28^{\circ} \mathrm{C} ;[\mathrm{DOC}]_{0}=10 \mathrm{mg} / \mathrm{L}$ )

Fig. 8 Effect of dissolved oxygen concentration on the photodegradation of A-DOM ( $500 \mathrm{~W}$ mercury lamp; without filtered membrane; $\mathrm{pH}=8.0 ; \mathrm{T}=28^{\circ} \mathrm{C} ;[\mathrm{DOC}]_{0}=10 \mathrm{mg} / \mathrm{L}$ ) 
生成的活性氧物质 (reactive oxygen species, ROS) 的氧化作用; 水中存在的中间介质吸收光子经过电子转移 过程将能量传递给有机质, 激发态的有机质和氧气反应生成 $\operatorname{ROS}^{[32-33]}$. 可见, DO 的存在对间接光解起到决 定性作用, 除去 DO, 有机质只能通过直接光解途径被降解. 对 C1、C2、C3 组分降解进行对比发现, C3 组分表 现出较小的差异, 说明直接光解 (通 $\mathrm{N}_{2}$ ) 能很好地降解 $\mathrm{C} 3$; 而间接光解 (通 $\mathrm{O}_{2}$ ) 对 $\mathrm{C} 3$ 的降解影响很小. DO 对难降解组分 $\mathrm{C} 1$ 和 C2 有很好的促进作用,间接光解是 C1 和 C2 的重要降解途径.

\section{4 结论}

1) 本文通过 A-DOM 光化学降解, 建立 EEMs-PARAFAC 组分模型, 鉴定 A-DOM 光化学降解过程中出现 的 4 个菼光组分: C1 ( UVC 类腐殖质)、C2 ( UVA 类腐殖质)、C3 (类色氨酸)、C4 (类络氨酸); 其对总苂光强 度的贡献分别为 $8.6 \% 、 22.2 \% 、 68.1 \%$ 和 $1.1 \%$.

2) 当以 $500 \mathrm{~W}$ 中压录灯为光源时, $\mathrm{pH}=8.0$, 反应温度为 $28^{\circ} \mathrm{C}$, 光照 $12 \mathrm{~h}$ 后 $a_{355}$ 降解率为 $70.4 \%$, 随着光 照时间的增加, A-DOM 的吸光度不断下降且分子量逐渐减小. 各 EEMs-PARAFAC 组分的光反应性为 C3> C1 $>$ C2.

3) 紫外光、光强、DO 的增加均能加速 A-DOM 的光化学降解. 对于较难降解的 C1 和 C2 组分, 可见光不 能使其降解, 其只能在紫外光下降解, 而 C3 组分在可见光和紫外光区都能降解; 光强增加对 3 个组分的降 解均有促进作用, 但降解速率的增加倍数小于光强增加的倍数; DO 的引人能很好地促进 C1 和 C2 的降解. 因此, 在消除蓝藻暴发产生的大量 A-DOM 以防止对饮用水安全产生危害时, 可结合实际情况, 选择外加紫 外光源、通人 DO 或者增加光源强度等手段来加速 A-DOM 的降解.

\section{5 参考文献}

[ 1 ] Li K, Xing B, Torello WA. Effect of organic fertilizers derived dissolved organic matter on pesticide sorption and leaching. Environmental Pollution, 2005, 134(2) : 187-194. DOI: 10.1016/j.envpol.2004.08.011.

[ 2 ] Yang M, Yu J, Li Z et al. Taihu Lake not to blame for Wuxi’s Woes. Science, 2008, 319(5860) : 158. DOI: 10.1371/ journal.phone.0051976.

[ 3 ] Stedmon CA, Markager S. Tracing the production and degradation of autochthonous fractions of dissolved organic matter by fluorescence analysis. Limnology and Oceanography, 2005, 50(5) : 1415-1426. DOI: 10.4319/lo.2005.50.5.1415.

[ 4 ] Stedmon CA, Markager S. Resolving the variability in dissolved organic matter fluorescence in a temperate estuary and its catchment using PARAFAC analysis. Limnology and Oceanography, 2005, 50(2) : 686-697. DOI: 10.4319/lo.2005.50. 2.0686 .

[ 5 ] Gao J, Jiang T, Yan JL et al. Photobleaching of dissolved organic matter (DOM) from confluence of two rivers under natural solar radiation: A case study of Fujiang River-Jialingjiang River. Environmental Science, 2014, 35(9) : 3397-3407. DOI : 10.13227/j.hjkx.2014.09.021. [高洁, 江蹈, 问金龙等. 天然日光辐照下两江交汇处溶解性有机质 (DOM) 光 漂白过程: 以涪江一一嘉陵江为例. 环境科学, 2014, 35(9): 3397-3407.]

[6 ] Du Y, Zhang Y, Chen F et al. Photochemical reactivities of dissolved organic matter ( DOM) in a sub-alpine lake revealed by EEM-PARAFAC: An insight into the fate of allochthonous DOM in alpine lakes affected by climate change. Science of the Total Environment, 2016, 568: 216-225. DOI: 10.1016/j.scitotenv.2016.06.036.

[ 7 ] Xu H, Jiang H. UV-induced photochemical heterogeneity of dissolved and attached organic matter associated with cyanobacterial bloomsina eutrophic freshwater lake. Water Research, 2013, 47 (17) : 6506-6515. DOI: 10.1016/j. watres. 2013.08.021.

[ 8 ] Zhang Y, Liu M, Qin B et al. Photochemical degradation of chromophoric-dissolved organic matter exposed to simulated UV-B and natural solar radiation. Hydrobiologia, 2009, 627(1) : 159-168. DOI: 10.1007/s10750-009-9722-z.

[ 9 ] Zhang Y, Hu CM, Yu T. Photodegradation of chromophoric dissolved organic matters in the water of Lake Dianchi, China. Frontiers of Environmental Science and Engineering, 2014, 9(4) : 575-582. DOI: 10.1007/s11783-014-0664-y.

[10] Guo WD, Cheng YY. Photodegradation of chromophoric dissolved organic matter from Jiulong River Estuary under natural solar radiation. Environmental Science, 2008, 29(6): 1463-1468. [郭卫东, 程远月. 天然日光辐照下河口区 CDOM 的光化学降解. 环境科学, 2008, 29(6): 1463-1468.]

[11] Stedmon CA, Markager S, Tranvik L et al. Photochemical production of ammonium and transformation of dissolved organic matter in the Baltic Sea. Marine Chemistry, 2007, 104(3/4) : 227-240. DOI: 10.1016/j.marchem.2006.11.005.

[12] Zhang YL, Liu XH, Osburn CL et al. Photobleaching response of different sources of chromophoric dissolved organic mat- 
ter exposed to natural solar radiation using absorption and excitation-emission matrix spectra. PLoS One, 2013, 8 (10): e77515.

[13] Gao HZ, Zepp RG. Factors influencing photoreactions of dissolved organic matter in a coastal river of the southeastern United States. Environmental Science and Technology, 1998, 32(19) : 2940-2946. DOI: 10.1021/es9803660.

[14] Hefner KH, Fisher JM, Ferry JL. A multifactor exploration of the photobleaching of Suwannee River dissolved organic matter across the freshwater/saltwater interface. Environmental Science and Technology, 2006, 40(12) : 3717-3722.DOI: 10. $1021 / \mathrm{es} 052513 \mathrm{~h}$.

[15] Lalonde K, Mucci A, Ouellet A et al. Preservation of organic matter in sediments promoted by iron. Nature, 2012 , 483 (7388) : 198-200. DOI: 10.1038/nature10855.

[16] Vecchio RD, Blough NV. Photobleaching of chromophoric dissolved organic matter in natural waters: Kinetics and modeling. Marine Chemistry, 2002, 78(4) : 231-253. DOI: 10.1016/S0304-4203(02)00036-1.

[17] Keith DJ, Yoder JA, Freeman SA. Spatial and temporal distribution of coloured dissolved organic matter ( CDOM) in Narragansett Bay, Rhode Island: Implications for phytoplankton in coastal waters. Estuarine Coastal and Shelf Science, 2002, 55(5) : 705-717. DOI: 10.1006/ecss.2001.0922.

[18] Helms JR, Stubbins A, Ritchie JD et al. Absorption spectral slopes and slope ratios as indicators of molecular weight, source, and photobleaching of chromophoric dissolved organic matter. Limnology and Oceanography, 2007, 53(3) : 955969. DOI: 10.4319/lo.2008.53.3.0955.

[19] Xiao YH, Timo SA, HelinÄ H et al. Contribution of ferric iron to light absorption by chromophoric dissolved organic matter. Limnology and Oceanography, 2013, 58(2) : 653-662. DIO : 10.4319/lo.2013.58.2.0653.

[20] Zhang YL, Zhang E, Yin Y et al. Characteristics and sources of chromophoric dissolved organic matter in lakes of the Yungui Plateau, China, differing in trophic state and altitude. Limnology and Oceanography, 2010, 55 (6): 2645-2659. DOI: $10.4319 / 10.2010 .55 .6 .2645$.

[21] Mcknight DM, Boyer EW, Westerhoff PK et al. Spectrophotometric characterization of dissolved organic matter for indication of precursor organic material and aromaticity. Limnology and Oceanography, 2001, 46(1): 38-48.

[22] Stedmon CA, Bro R. Characterizing dissolved organic matter fluorescence with parallel factor analysis: A tutorial. Limnology and Oceanography Methods, 2008, 6(11) : 572-579. DOI: 10.4319/lom.2008.6.572.

[23] Guo WD, Cheng YY. Photodegradation of chromophoric dissolved organic matter from Jiulong River Estuary under natural solar radiation. Environmental Science, 2008, 29(6) : 1463-1468. [郭卫东, 程远月. 天然日光辐照下河口区 CDOM 的光化学降解. 环境科学, 2008, 29(6): 1463-1468.]

[24] Liu X, Zhang Y, Yin Y et al. Wind and submerged aquatic vegetation influence bio-optical properties in large shallow Lake Taihu, China. Journal of Geophysical Research Biogeosciences, 2013, 118(2) : 713-727. DOI: 10.1002/jgrg.20054.

[25] Osburn CL, Handsel LT, Mikan MP et al. Fluorescence tracking of dissolved and particulate organic matter quality in a river-dominated estuary. Environmental Science and Technology, 2012, 46(16) : 8628-8636. DOI: 10.1021/es3007723.

[26] Murphy KR, Ruiz GM, Dunsmuir WTM et al. Optimized parameters for fluorescence-based verification of ballast water exchange by ships. Environmental Science and Technology, 2006, 40(7) : 2357-2362. DOI : 10.1021/es0519381.

[27] Cory RM, Mcknight DM. Fluorescence spectroscopy reveals ubiquitous presence of oxidized and reduced quinones in dissolved organic matter. Environmental Science and Technology, 2005, 39(21): 8142-8149. DOI: 10.1021/es0506962.

[28] Cheng YY, Guo WD. Characterization of photobleaching of chromophoric dissolved organic matter in Xiamen bay by excitation emission matrix spectroscopy. Spectroscopy and Spectral Analysis, 2009, 29(4) : 990-993.

[29] Sommaruga R. The role of solar UV radiation in the ecology of alpine lakes. J Photochem Photobiol B, 2001, 62( 1/2) : 35-42. DOI : 10.1016/S1011-1344(01)00154-3.

[30] Kieber RJ, Zhou X, Mopper K. Formation of carbonyl compounds from UV-induced photodegradation of humic substances in natural waters: Fate of riverine carbon in the sea. Limnology and Oceanography, 1990, 35(7): 1503-1515. DOI: 10. 4319/lo.1990.35.7.1503.

[31] Yang DT, Chen WM, Wu SC et al. The effects of CDOC on the attenuation of close ultraviolet and blue light in Chaohu Lake and Longgan Lake. J Lake Sci, 2003, 15(3) : 269-274. DOI: 10.18307/2003.0312. [杨顶田，陈伟民，吴生才 等. 湖泊中有色可溶性物质对近紫外及蓝光衰减的影响. 湖泊科学, 2003, 15(3): 269-274.]

[32] Cory RM, Mcneill K, Cotner JP et al. Singlet oxygen in the coupled photochemical and biochemical oxidation of dissolved organic matter. Environmental Science and Technology, 2010, 44(10) : 3683-3689. DOI: 10.1021/es902989y.

[33] Song XN, Yu T, Zhang Y et al. Distribution characterization and source analysis of dissolved organic matters in Taihu Lake using three dimensional fluorescence excitation-emission matrix. Journal of Environmental Sciences, 2010,30 ( 11) : 23212331. [ 宋晓娜, 于涛, 张远等. 利用三维苂光技术分析太湖水体溶解性有机质的分布特征及来源. 环境科学学 报, 2010, 30(11): 2321-2331.] 